\title{
La Influencia de la Implementación de las Tecnologías de Información en la Productividad de Empresas de Servicios
}

\author{
Danny Arévalo-Avecillas ${ }^{(1) \star}$, Santiago Nájera-Acuña ${ }^{(1)}$ y Edgar A. Piñero(2) \\ (1) CENTRUM Católica Graduate Business School, Jr. Daniel Alomía Robles 125, Urb. Los Álamos de \\ Monterrico, Pontificia Universidad Católica del Perú, Lima, Perú. \\ (2) Universidad Técnica de Manabí, Avenida Jose Maria Urbina, Portoviejo, Ecuador. \\ (e-mail: danny.arevalo@pucp.pe; snajera072@puce.edu.ec; alexanderpinero12@gmail.com)
}

${ }^{*}$ Autor a quien se debe dirigir de correspondencia

Recibido Mar. 19, 2018; Aceptado May. 24, 2018; Versión final Jun. 22, 2018, Publicado Dic. 2018

\begin{abstract}
Resumen
La investigación tiene como propósito determinar la influencia de las tecnologías de la información en la productividad de las empresas de servicios, considerando el efecto moderador de las variables explicativas inversión en capacitación, inversión en gestión del conocimiento y las prácticas administrativas. El estudio es no experimental, de alcance correlacional-explicativo y de enfoque cuantitativo. Se utilizaron fuentes de datos secundarios, obtenidos por medio de bases de datos oficiales, desde el año 2010 hasta el 2016. El estudio presenta un análisis de datos de panel a partir de una función de producción de Cobb-Douglas y modelos de regresión para el análisis de largo plazo. Los resultados demuestran que la inversión en tecnologías de la información influye positivamente en la productividad y que la gestión del conocimiento cumple el rol de variable moderadora en el corto y largo plazo.
\end{abstract}

Palabras clave: tecnologías de información; productividad; productividad innovadora; empresas de servicios; datos de panel; gestión del conocimiento

\section{The Influence of the Implementation of Information Technologies in the Productivity of Service Companies}

\begin{abstract}
The research aims to determine the influence of information technology on productivity of service companies, considering the moderating effect of investment in training, investment in knowledge management and administrative practices. The study is non-experimental, with correlation-explanatory scope and quantitative approach. Primary data was obtained through official databases from 2010 to 2016 . The study presents an analysis of panel data from a Cobb-Douglas production function and regression models for the long-term analysis. Results show that investing in information technology positively influences productivity and knowledge management plays the role of a moderating variable in short and long-term.
\end{abstract}

Keywords: information technologies; productivity; innovative productivity; service companies; panel data; knowledge management 


\section{INTRODUCCIÓN}

En la era actual las tecnologías de información ( $\mathrm{TI}$ ) están evolucionando a un ritmo vertiginoso. Países desarrollados como Estados Unidos, Canadá, Japón, Suecia, Finlandia, Noruega, Corea del Sur, Suiza, entre otros, utilizan las tecnologías de información para generar procesos más sistematizados y mejorar el desempeño de sus industrias (Arvanitis y Loukis, 2015; Black y Lynch, 2001). De hecho, el World Economic Forum (WEF, 2014) reconoció que un 10\% de uso de internet de banda ancha generó un incremento del $0.25 \%$ del PIB y que los países con mayor uso de TI experimentan una productividad empresarial siete veces mayor que los países que no invierten de forma apropiada en TI. Por otro lado, los servicios representan un sector muy importante de la economía, precisamente por su sofisticación y alto valor agregado. Inclusive, estudios señalan que un aumento o mejora de la productividad de este sector impacta positivamente en los niveles de vida de varios países (Krugman y Obstfeld, 2006), o bien constituye en un factor fundamental en el desarrollo de la sociedad (Afflerbach, 2015; Brynjolfsson y Hitt, 1996; Liu et al., 2013). De acuerdo a la Organización Mundial del Comercio (OMC, 2017), en América Latina, los servicios representaron aproximadamente el 64\% del valor añadido en el 2016, muy por encima del $49 \%$ que se registró hace 40 años atrás. Estas firmas usan las tecnologías de información para incrementar sus competencias gerenciales por medio del uso de herramientas y plataformas tecnológicas.

Las TI contribuyen al desarrollo del potencial y a la mejora de los niveles de productividad de las empresas mediante la sistematización y optimización de sus procesos (Orlikowski y lacono, 2001; Zwick, 2003). Las TI ayudan a las empresas a mejorar el flujo información adecuada para la correcta toma de decisiones y a perfeccionar los procesos internos de comunicación en las firmas (Afflerbach, 2015; Cardona et al., 2013). Aral et al. (2012) indicaron que mejorar el rendimiento y la eficiencia dentro de las organizaciones se ha convertido en un tema complejo, por la dificultad de su aplicación, causado por la improvisación e intercambio de la información sobre métodos, manuales o evaluación previa de las diversas actividades gerenciales u organizacionales (Alavi y Leidner, 2001). Por este motivo, el efecto de las TI produce un flujo de información eficaz para generar una mayor descentralización y permitir al personal tomar decisiones (Dedrick y Kraemer, 2001; Ghobakhloo et al., 2012).

\section{Fundamentación Teórica}

Cobo (2009) define el término tecnologías de información (TI) como dispositivos electrónicos de computación (hardware y software), telecomunicaciones, microelectrónica y optoelectrónica que permiten el procesamiento y acumulación de enormes cantidades de información, además de una rápida distribución de la información a través de redes de comunicación. Siguiendo esta definición, las TI pueden ser medidas de dos formas: (a) por medio de datos duros tomando en consideración el monto de inversión que destinan las empresas a la adquisición de tecnología (Brynjolfsson y Hitt, 1996; Dedrick et al., 2011; Sabherwal y Jeyaraj, 2015); (b) por medio de datos cualitativos que analicen el comportamiento de los sistemas de información y su influencia en el éxito de las organizaciones (Delone y McLean, 2003). Durante los últimos años, varios estudios han analizado el impacto empresarial de las TI (Aral et al., 2012). En los años 90, existían teorías asociadas principalmente a la paradoja de la productividad de Robert Solow (Brynjolfsson y Hitt, 2003) y se formularon modelos estadísticos para analizar la relación entre las inversiones en TI y la productividad (Arévalo-Avecillas et al., 2017; Black y Lynch, 2001; Ghobakhloo et al., 2012). Hitt y Brynjolfsson (1996) encontraron que las firmas que implementan TI y descentralizan sus operaciones son cinco por ciento más productivas. Según Dedrick et al. (2011), las TI tienen un efecto positivo en la productividad de las empresas en países desarrollados, principalmente porque existe descentralización en la toma de decisiones y las TI se encargan de viabilizar los diversos procesos en las firmas (Cardona et al., 2013). Por el contrario, las TI en los países en vías de desarrollo poseen poca influencia en la productividad de las firmas, puesto que la mayoría de las decisiones de las firmas aún son centralizadas (Peng et al., 2016). Además, Licht y Moch (1999) indicaron que la inversión en TI tiene una influencia positiva sobre la productividad en las empresas de servicios.

Varios estudios miden la productividad por medio del total de ventas o ingresos generados en un período determinado (Biege et al., 2013; Djellal y Gallouj, 2008), o a través de ratios de ventas para el total de trabajadores (Black y Lynch, 2001). Brynjolfsson y Hitt (2003) asienten a esta premisa, indicando que las inversiones en TI pueden producir beneficios en la productividad. No obstante, su incremento depende tanto de la innovación tecnológica como de los cambios organizacionales realizados por la firma (Banker y Natajaram, 2008). Hwang et al. (2015) señalaron también que las TI influyen en la productividad por medio de la generación e innovación de nuevos servicios. Además, Chang y Gurbaxani (2012) realizaron un estudio en un número reducido de países en vías de desarrollo y evidenciaron relaciones poco relevantes entre la inversión en TI y la productividad de las firmas, puesto que, por cada dólar que se invierte en TI, es necesario invertir hasta diez dólares en otras áreas estratégicas para alcanzar los mismos resultados en términos de productividad en comparación con países desarrollados (Brynjolfsson y Hitt, 1996; Chen y Tsou, 2012). 
Con base en estos argumentos, Klomp y Van Laeeuwen (2001) sugieren el análisis de la productividad innovadora, definida como el nivel de ventas generadas por innovaciones nuevas para el mercado. De esta forma, se eliminan problemas de sesgo relacionados con otras causas que influyen en la productividad. Los autores marcan que los valores de la productividad innovadora están asociados a resultados innovadores tangibles, producto de la inversión en infraestructura tecnológica que proporciona ventajas económicas a las organizaciones. Las innovaciones nuevas en el mercado hacen referencia a innovaciones incrementales, que consiste en una mejora de los servicios existentes y a innovaciones radicales, que conllevan a la exploración y explotación de nuevos mercados (Cefis y Ciccarelli, 2005). Otros estudios también afirman que la rentabilidad puede ser medida bajo el mismo enfoque, por medio del total de utilidades generadas por nuevos servicios o innovaciones en el mercado (Cefis y Ciccarelli, 2005) y respaldan la relación entre la inversión en tecnologías de información y la rentabilidad (Djellal y Gallouj, 2008; Ong y Chen, 2013).

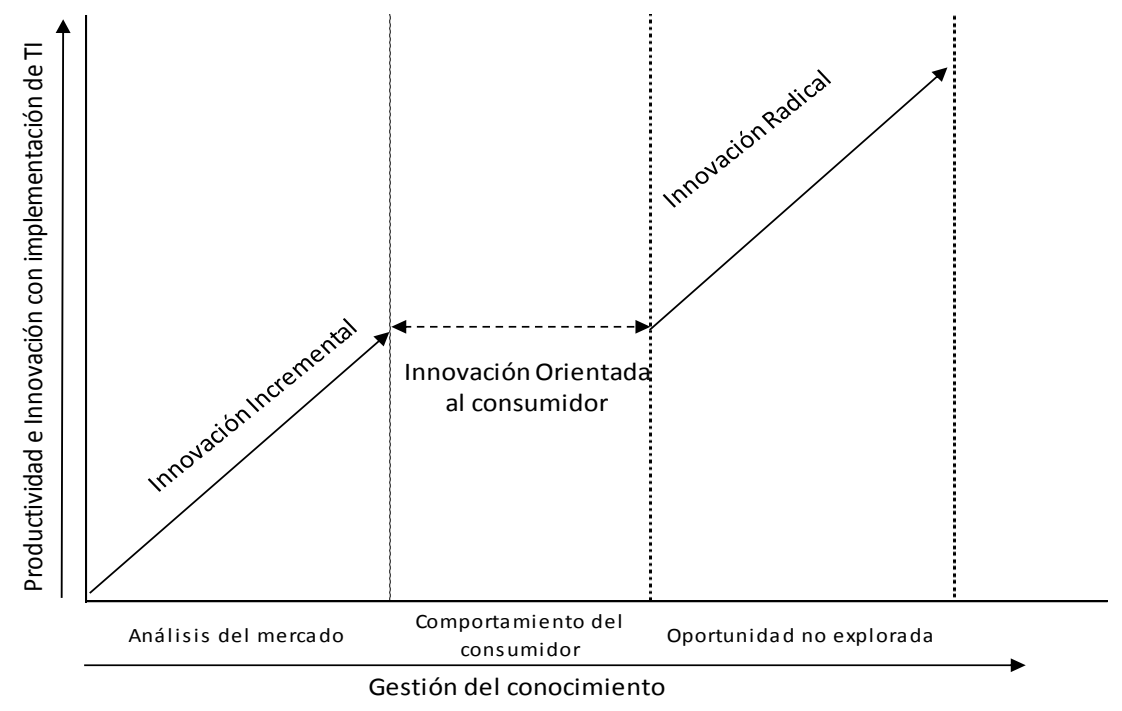

Fig. 1: Relación de la gestión del conocimiento con la productividad e innovación en las firmas de servicios

Diversas investigaciones han señalado que la inversión e implementación de las $\mathrm{TI}$ no incrementa la productividad automáticamente (Afflerbach, 2015; Brynjolfsson y Hitt, 2003). Este efecto ocurre principalmente por la falta de integración de las TI en la estructura organizacional, en donde se debe dar atención al nivel de capacitación de los empleados, gestión eficiente del conocimiento y mejora continua en las prácticas administrativas de las firmas (Alavi y Leidner, 2001; Bertschek et al., 2013; Sabherwal y Jeyaraj, 2015). Según Lichtenberg (1995), un empleado bien calificado, que trabaje con tecnologías de información, puede ser más eficiente que seis trabajadores que no conozcan cómo manejar y elaborar procesos con TI. De hecho, numerosos estudios justifican que no es suficiente el aporte de las TI en la productividad, porque el éxito de la firma se encuentra en función de personas capacitadas en manejo de tecnologías (Dedrick y Kraemer, 2001; Dewan y Kraemer, 2000; Hawash y Lang, 2010; Pierce et al., 2015). Zwick (2003) argumentó que existen variables como, por ejemplo, la inversión en entrenamiento o capacitación continua del personal que puede mejorar la productividad y rentabilidad de las organizaciones. Por consiguiente, el creciente nivel de competitividad en los mercados ha creado la necesidad de capacitar al personal de las firmas de forma constante (Aral et al., 2012; Arzola y Mejias, 2010), en búsqueda de una mayor productividad, así como procesos de innovación más sofisticados.

Dedrick y Kraemer (2001) afirmaron que la gestión del conocimiento es un componente importante en las empresas de servicios. La suma del conocimiento y destrezas de las firmas determinan la habilidad para generar servicios, ganando aceptación en el mercado y logrando éxito financiero (Alavi y Leidner, 2001). De esta forma, la empresa evoluciona en el mercado a través de un eficiente manejo del flujo de conocimiento para generar productividad e innovación (Chuang et al., 2013; Marulanda et al., 2013). Adicionalmente, Biege et al. (2013) indica que una productividad sostenida en el tiempo es alcanzable a través de la intensidad y manejo del conocimiento, que se convierte en un input crucial para generar procesos productivos renovados en el mediano y largo plazo. Licht y Moch (1999) sugirieron medir la gestión del conocimiento a través de la inversión en investigación y desarrollo de las empresas. Esto es ratificado por Brynjolfsson y Hitt (1996), que proponen cuantificar el total de gastos de las firmas o el porcentaje destinado a investigación y desarrollo. Además, Ortega et al. (2014) determinaron que la gestión del conocimiento es medida con la inversión en investigación y desarrollo y que dicha variable tiene un impacto significativo en la productividad. 
Por otro lado, uno de los retos más importantes es direccionar las TI a las prácticas administrativas para generar productividad y un mejor desempeño en las organizaciones (Afflerbach, 2015). Según Ichniowski et al. (1997) las prácticas administrativas innovadoras tienen una relación importante en la productividad de las organizaciones. Por consiguiente, cuando las firmas invierten y gestionan de forma eficiente las prácticas laborales y administrativas, la implementación de las tecnologías de información y la inversión en capital humano capacitado, ocurre un impacto relevante en la productividad (Brynjolfsson y Hitt, 2003; Devaraj y Kohli, 2003; Zwick, 2003). En este contexto, no es suficiente analizar la aplicación de las TI en las empresas y el uso que se les da (Orlikowski y lacono, 2001), sino también, es importante generar procesos de integración con las prácticas administrativas de las firmas, en búsqueda de un mejor desempeño y productividad (Charoensuk et al., 2014; Kraemer y Dedrick, 2001; Tallon et al., 2000).

Los escenarios a continuación indican cómo la falta de integración de las TI afecta a la estructura organizacional de las firmas: (a) errores en la medición de capital de TI, debido a los cambios rápidos y vertiginosos en los precios, en la calidad de las TI, (b) retrasos de tiempo, es decir, las tecnologías de información no tienen un impacto medible en la productividad hasta alcanzar una masa crítica de difusión y experiencia en la organización, (c) prácticas administrativas, que no han evolucionado lo suficiente para aprovechar al máximo el potencial de las tecnologías de información y (d) redistribución, es decir, las TI podrían ayudar a las firmas en relación a sus competidores, pero no incrementar la productividad de toda la economía (Aral et al., 2012; Brynjolfsson y Hitt, 1996; Cao y Hoffman, 2011). Por consiguiente, la inversión en TI tendría un impacto positivo en la productividad de las firmas a partir de una integración eficiente de las TI, capacitación adecuada del personal, generación de investigación y desarrollo sostenible y mejoras en las prácticas administrativas (Alavi y Leidner, 2001; Cao y Hoffman, 2011; Dedrick y Kraemer, 2001; Ghobakhloo et al., 2012; Sabherwal y Jeyaraj, 2015; Rawley y Simcoe, 2013).

Luego de una revisión teórica de las variables explicativas inversión en capacitación, inversión en gestión del conocimiento y las prácticas administrativas, algunos autores sugieren que dichas variables pueden moderar la relación entre las TI y la productividad (Alavi y Leidner, 2001; Arzola y Mejias, 2010; Brynjolfsson y Hitt, 2003; Darroch y McNaughton, 2002; Dedrick y Kraemer, 2001; Licht y Moch, 1999). La implementación y el uso de las TI permite realizar cambios radicales en la estructura organizacional de las empresas (Cao y Hoffman, 2011), a través de la alineación de las tecnologías de información a las prácticas administrativas y mejora de sus procesos para la toma de decisiones, aprovechando de esta forma el dinamismo de las $\mathrm{TI}$ (Dedrick y Kraemer, 2001; Rawley y Simcoe, 2013). De igual modo, la capacitación constante del talento humano en manejo de TI permite a los empleados pasar de una tarea a otra de manera rápida y desarrollar con mayor facilidad su trabajo (Orlikowski y lacono, 2001), aumentando el desempeño y contribuyendo así con los indicadores de productividad de la compañía (Brynjolfsson y Hitt, 1996; Dedrick y Kraemer, 2001). Finalmente, la implementación de las TI permite a las organizaciones ser productivas y competitivas en el mediano y largo plazo (Lapointe y Rivard, 2005; Melville, 2001), debido a que su éxito se da en función de las personas capacitadas en el manejo de recursos tecnológicos. De esta forma, las firmas logran una diferenciación en sus procesos internos y generan un manejo adecuado de la gestión del conocimiento (Afflerbach, 2015; Alavi y Leidner, 2001; Dedrick y Kraemer, 2001; Licht y Moch, 1999; Hawash y Lang, 2010).

Bajo este contexto, el propósito de la investigación consiste en determinar la influencia de las TI en la productividad de las empresas de servicios, teniendo en consideración el efecto moderador de la inversión en capacitación, inversión en gestión del conocimiento y las prácticas administrativas, variables ampliamente reconocidas en la literatura (Alavi y Leidner, 2001; Black y Lynch, 2001; Hitt y Brynjolfsson, 1996; Liu et al., 2013). Considerando este propósito se establece las siguientes preguntas de investigación: (a) ¿Tiene la inversión en tecnologías de información influencia en la productividad de las empresas de servicios? (b) ¿Las prácticas administrativas, la gestión del conocimiento y la capacitación adoptan el rol de variables moderadoras entre la relación de la inversión en TI y la productividad en las empresas de servicios? y (c) ¿Tiene la inversión en tecnologías de información influencia en la rentabilidad de las empresas de servicios?

En el presente estudio se formulan las siguientes hipótesis: (a) $\mathrm{H}_{1}$ : La inversión en TI influye en la productividad de las empresas de servicios en el corto y largo plazo; (b) $\mathrm{H}_{2}$ : Las prácticas administrativas, la inversión en gestión del conocimiento y la inversión en capacitación cumplen el rol de variables moderadoras entre la relación de la inversión en TI y la productividad en las empresas de servicios en el corto y largo plazo. Adicionalmente se plantea una nueva hipótesis $\mathrm{H}_{3}$ : La inversión en TI influye en la rentabilidad de las empresas de servicios en el largo plazo.

\section{METODOLOGÍA}

La investigación es no experimental, de alcance correlacional y explicativo, de lógica deductiva y de enfoque cuantitativo (Hernández et al., 2010). La investigación es de corte transversal y se utilizaron fuentes de datos secundarios. Con base en la revisión de literatura e hipótesis de investigación, el estudio presenta seis 
variables: (a) inversión en TI, (b) inversión en capacitación, (c) prácticas administrativas, (d) inversión en gestión del conocimiento, (e) productividad innovadora y (f) rentabilidad innovadora, estas dos últimas representan la variable endógena del modelo. Los datos secundarios corresponden a información de las bases de datos de la Superintendencia de Compañías del Ecuador, desde el año 2010 hasta el 2016.

Las TI fueron medidas a través de la inversión en tecnologías de información que realizan las firmas del sector de servicios (Brynjolfsson y Hitt, 1996; Chang y Gurbaxani, 2012; Dedrick et al., 2011; Sabherwal y Jeyaraj, 2015). La medición de las variables moderadoras capacitación y gestión del conocimiento se llevó a cabo por medio de los montos de inversión que destinan las empresas de servicios a la capacitación del talento humano (Hawash y Lang, 2010; Pierce et al., 2015; Zwick, 2003) e investigación y desarrollo (Licht y Moch, 1999; Ortega et al., 2014). La productividad innovadora fue medida por medio del total de facturación o ventas generadas por nuevos servicios en el mercado entre el número de trabajadores y la rentabilidad innovadora representa el total de utilidad procedente de nuevos servicios en el mercado entre el número de trabajadores (Biege et al., 2013; Cefis y Ciccarelli, 2005, Djellal y Gallouj, 2008; Klomp y Van Leeuwen, 2001). Se utilizó además escalas binarias para evaluar la integración de las TI en las prácticas administrativas de las firmas (Arvanitis y Loukis, 2015; Dedrick y Kraemer, 2001; Ortega et al., 2014).

\section{Población}

La población de estudio está conformada por las empresas pertenecientes al sector de servicios a nivel urbano, ya que existe una gran diversificación de estratos, como el de consultoría, inmobiliarias, transporte, servicios sociales y de salud, alojamiento, actividades de servicios financieros, entre otros. En términos de requisitos, las empresas de servicios participantes deben tener un tiempo de operación mínimo de tres años y que, al momento de la investigación, se encuentren como activas en la superintendencia de compañías del Ecuador. La población de las empresas de servicios de los estratos considerados es de 18,320 firmas, sin embargo, las empresas debían presentar nuevos servicios o innovaciones en el mercado en alguno de los últimos siete años (2010-2016), debido al enfoque que se da a la variable endógena productividad innovadora. Con base en este antecedente, la población considerada en el estudio es de 11,115 empresas de servicios.

\section{Marco Muestral}

Se consideró los cinco estratos más representativos del sector de servicios, que aportan en más de 3,000 millones de dólares a dicho sector: (a) actividades profesionales, técnicas y administrativas (consultoras), (b) inmobiliarias, (c) transporte y almacenamiento, (d) servicios sociales y de salud y (e) actividades de servicios financieros. Estas empresas debían tener un tiempo de operación mínimo de tres años y presentar una inversión mínima en TI del $1 \%$ de las ventas. La técnica de muestreo aleatorio sistemático fue empleada en el estudio con la finalidad de que cada firma se encuentre representada en la muestra seleccionada (Hernández et al., 2010; Malhotra, 2008). Se aplicó la fórmula para poblaciones finitas, con una probabilidad de éxito del $50 \%$, un margen de error del cinco por ciento y un nivel de confianza del $95 \%$. El resultado proyectó una muestra aproximada de 375 empresas, divididas equitativamente para cada estrato. El estudio se desarrolló en las ciudades de Guayaquil y Quito por albergar el mayor porcentaje de empresas que ofertan servicios en Ecuador.

\section{Análisis de Datos}

El estudio presenta un análisis de datos de panel en primera instancia, con la finalidad de determinar la influencia de la inversión en tecnologías de información (TI) y su interacción con las variables inversión en capacitación (CA), gestión del conocimiento (GC) y prácticas administrativas (PA) en la productividad y rentabilidad innovadora de las firmas de servicios (Afflerbach, 2015). La literatura además recomienda trabajar con ratios, por lo que la variable de control tamaño o número de trabajadores fue considerada para las variables continuas del modelo (Black y Lynch, 2001; Klomp y Van Laeeuwen, 2001; Licht y Moch, 1999). Para efectos del análisis de datos de panel se estimó una función de producción de Cobb-Douglas (Black y Lynch, 2001; Brynjolfsson y Hitt, 2003; Hawash y Lang, 2010). Dicha función incluye como variables la inversión en tecnologías de información (TI), inversión en capacitación $(\mathrm{CA})$, inversión en gestión del conocimiento (GC), prácticas administrativas (PA) y número de trabajadores (L). y como variable dependiente la productividad innovadora (ventas de nuevos servicios en el mercado por número de trabajadores).

El estudio reporta tres modelos para el análisis de datos de panel con efectos fijos en los agentes y en los agentes - tiempo. Como la especificación básica Cobb-Douglas no es lineal, se aplicaron logaritmos en la ecuación más la adición de un término de error (Brynjolfsson y Hitt, 2003). La especificación general de los tres modelos propuestos con datos de panel es la siguiente: 


$$
\begin{aligned}
& \Delta \ln Y_{i, t}=\beta_{i t}+\beta_{1} \Delta \ln (T I / L)_{i, t}+\beta_{2} \Delta \ln (C A / L)_{i, t}+\beta_{3} \Delta \ln (G C / L)_{i, t}+\beta_{4}(P A)_{i, t}+U_{i t} \\
& \begin{array}{ll}
\Delta \ln Y_{i, t}=\beta_{i t}+\beta_{1} \Delta \ln (T I / L)_{i, t}+\beta_{2} \Delta \ln (T I / L)_{i, t} * \Delta \ln (C A / L)_{i, t}+\beta_{3} \Delta \ln (T I / L)_{i, t} * \Delta \ln (G C / L) i, t+ \\
\beta_{4} \Delta \ln (T I / L)_{i, t} *(P A)_{i, t}+U_{i t}
\end{array} \\
& \begin{array}{l}
\Delta \ln Y_{i, t}=\beta_{i t}+\beta_{1} \Delta \ln (T I / L)_{i, t}+\beta_{2} \Delta \ln (C A / L)_{i, t}+\beta_{3} \Delta \ln (G C / L)_{i, t}+\beta_{4}(P A)_{i, t}+\beta_{5} \Delta \ln (T I / L)_{i, t} * \Delta \ln (C A / L)_{i, t}+ \\
\beta_{6} \Delta \ln (T I / L)_{i, t} * \Delta \ln (G C / L)_{i, t}+\beta_{7} \Delta \ln (T I / L)_{i, t} *(P A)_{i, t}+U_{i t}
\end{array}
\end{aligned}
$$

En estas ecuaciones, TI, CA, GC, PA y L son las variables del modelo; $\beta_{i t}$ es la constante del modelo, es decir el vector de intercepto; $t$ se refiere a la dimensión del tiempo e $i$ representa cada firma del sector de servicios.

La siguiente instancia de la investigación incluye un análisis de regresiones lineales para determinar el impacto de las tecnologías de información (TI) y las variables explicativas del estudio en la productividad y rentabilidad innovadora en el largo plazo, por medio de la tasa de crecimiento promedio anual (Arvanitis y Loukis, 2015; Black y Lynch, 2001; Dedrick y Kraemer, 2001). En este contexto, se calculó la diferencia entre el valor de inversión en tecnologías de información ( $\mathrm{TI} / \mathrm{L})$, capacitación $(\mathrm{CA} / \mathrm{L})$, gestión del conocimiento (GC/L), productividad y rentabilidad del año 2016 menos su equivalente al año 2010, dividido para el número de años del período considerado. El operador de todas las variables explicativas está denotado por $\Delta X_{10}{ }^{-16}=$ $X_{16}-X_{10}$. De igual modo, el operador de las variables dependientes está denotado por $\Delta Y_{10}{ }^{-16}=Y_{16}-Y_{10}$.

\section{RESULTADOS}

Para el desarrollo del primer análisis se tomó en cuenta los datos de las variables principales del modelo por ciudades y estratos. Entre los principales resultados se destaca que 206 firmas pertenecen a la ciudad de Quito y 169 a la ciudad de Guayaquil. En promedio, las firmas de servicios generaron durante el 2016 un ingreso por nuevos servicios de aproximadamente $\$ 568,326.96$ y una utilidad por nuevos servicios en el mercado de $\$ 116,298.48$, es decir un margen neto de $20.46 \%$. De esta información, Guayaquil es la ciudad que más ingresos y utilidades generadas presenta. Un análisis de diferencia de medias fue realizado como método de comprobación para determinar si existen diferencias significativas entre ciudades mediante el contraste de la prueba $\mathrm{F}$, donde la hipótesis alternativa denota desigualdad entre la media de las muestras independientes ( $H 1: \mu 1 \neq \mu 2$ ). Todos los casos exhibieron valores $p$ mayor a 0.05 ; por lo tanto, se concluye que no existen diferencias significativas de las variables analizadas entre ciudades y que pertenecen a una misma población.

Tabla 1: Prueba de Igualdad de Medias y Homogeneidad de Varianzas por Estrato

\begin{tabular}{|l|c|c|c|c|}
\hline \multicolumn{1}{|c|}{ Variables } & Prueba $F$ & Sig. & Estadístico de Levene & Sig. \\
\hline Inversión en TI/L & 9.944 & .000 & 18.431 & .000 \\
\hline Inversión en CA/L & 6.632 & .000 & 10.905 & .000 \\
\hline Inversión en GC/L & 10.961 & .000 & 13.033 & .000 \\
\hline Total de Ventas/L & 17.036 & .000 & 26.708 & .000 \\
\hline Utilidad Neta/L & 14.209 & .000 & 18.054 & .000 \\
\hline
\end{tabular}

Posteriormente fueron considerados los cinco estratos del estudio. Los resultados evidenciaron que los estratos de servicios sociales y de salud y actividades de servicio financiero generaron el mayor nivel de ventas y utilidades durante el 2016. Asimismo, las firmas de servicios sociales y de salud son quienes presentan mayor inversión en tecnologías de información, capacitación e investigación y desarrollo. De esta información, Guayaquil es la ciudad que más ingresos y utilidades generadas presenta. Se llevó a cabo el mismo contraste de medias, esta vez por estrato. Los resultados indican que todas las variables presentaron valores $p$ inferiores a 0.05 , por lo que se concluye que estas variables no cumplen con el supuesto de homogeneidad de las varianzas. Esto se da debido a que cada estrato posee sus propias características y maneja distintos montos de inversión en TI, capacitación e investigación y desarrollo.

\section{Prueba de raíces unitarias}

Adicionalmente se realizó una prueba de raíces unitarias de todas las variables del modelo para detectar estacionariedad (Kruiniger, 2013). Bajo escenarios de tendencia y cuando el panel está conformado por más agentes que series de tiempo, la literatura siguiere usar el test de Breitung, cuya hipótesis nula $\left(\mathrm{H}_{0}\right)$ expresa problemas de raíces unitarias y la hipótesis alternativa $\left(\mathrm{H}_{1}\right)$, estacionariedad de las variables, es decir que no 
presentan problemas de raíces unitarias. Las probabilidades para test de Breitung son computadas usando una distribución asintótica de Chi-cuadrado (Dhaene y Jochmans, 2016). La tabla 2 reporta el análisis de raíces unitarias con un rezago en niveles y con primera diferencia. En niveles, los resultados presentan valores p superiores a 0.05 para todas las variables, denotando problemas de raíces unitarias. Han y Phillips (2013) recomiendan la aplicación de primeras diferencias a las variables para revertir los efectos de raíces unitarias, es decir, restar un período anterior a cada variable $X t-X t-1 o Y t-Y t-1$. De este modo, $\Delta X t=X t-X t-1$ se define como el operador de primera diferencia para cada variable explicativa del modelo.

Tabla 2: Pruebas de Raíces Unitarias para las Variables Individuales del Modelo

\begin{tabular}{|l|c|c|c|c|c|}
\hline En niveles & $T I$ & $C A$ & $G C$ & PROD & RENT \\
\hline Con un rezago y con tendencia \\
\hline Breitung test & 2.187 & 0.639 & -0.426 & -0.063 & 0.167 \\
\hline$p$-value & 0.986 & 0.739 & 0.335 & 0.475 & 0.567 \\
\hline En primera diferencia & $\Delta \mathrm{TI}$ & $\Delta \mathrm{CA}$ & $\Delta \mathrm{GC}$ & $\Delta \mathrm{PROD}$ & $\Delta$ RENT \\
\hline Con un rezago y con tendencia \\
\hline Breitung test & -18.748 & -6.409 & -22.000 & -28.913 & -17.404 \\
\hline$p$-value & 0.000 & 0.000 & 0.000 & 0.000 & 0.000 \\
\hline
\end{tabular}

El nuevo análisis de las variables en primera diferencia demuestra que se han superado problemas de raíces unitarias. Finalmente, las pruebas de Barlett, Leven y Brown-Forsythe fueron empleadas en cada panel para detectar problemas de heterocedasticidad. Todos los paneles presentaron problemas de heterocedasticidad; debido a esto, el problema fue corregido aplicando la corrección de White (Pérez y López, 2008), donde los parámetros estimados no cambian; no obstante, existirán cambios en los errores estándar (Kruiniger, 2013).

\section{Análisis con datos de panel basado en la productividad}

En esta instancia se exhibe seis paneles donde la variable dependiente es la productividad innovadora de las firmas. Previo al análisis, se efectuó el análisis de normalidad univariante y multivariante. En el primer caso el rango intercuartílico fue calculado, mostrando resultados muy cercanos a 1.3 y denotando normalidad univariante. Para el segundo caso, el test de Henze-Zirkler fue calculado, donde la hipótesis nula expresa normalidad multivariante de los datos (Lantz et al., 2016). El test reveló un p-value de 0.127, por lo que la data utilizada posee normalidad multivariante. La tabla 3 presenta las estimaciones de los paneles A, B y C controlados por efectos fijos en los agentes. Por otra parte, los paneles D, E y F presentan las estimaciones controlados por efectos fijos en los agentes (firmas) y en el tiempo (año). Los números en paréntesis reportan el valor del error estándar y se consideraron niveles de significancia de $10 \%\left({ }^{*}\right), 5 \%\left({ }^{* \star}\right)$ y $1 \%\left({ }^{* * \star}\right)$.

En el panel A, los coeficientes de las variables tecnologías de información (TI/L), inversión en capacitación $(\mathrm{CA} / \mathrm{L})$ e inversión en gestión del conocimiento $(\mathrm{GC} / \mathrm{L})$ son significativos y positivos a un nivel de confianza del 99\%; mientras que el coeficiente de la variable prácticas administrativas (PA) es significativa al 95\% de confianza. Para mejor comprensión de los resultados, la derivada de cada variable $X_{j}$ ha sido obtenida con respecto a la productividad, definida en la siguiente expresión: $\beta j=\partial \Delta \ln Y / \partial \Delta \ln X j$. En este contexto, si la tasa de crecimiento de $\mathrm{Tl} / \mathrm{L}$ aumenta en un punto porcentual, entonces la tasa de crecimiento de la productividad innovadora $(\mathrm{Q} / \mathrm{L})$ aumenta en 0.3800 puntos porcentuales. Por otra parte, si la tasa de crecimiento de $\mathrm{GC} / \mathrm{L}$ aumenta en un punto porcentual, entonces la tasa de crecimiento de la productividad innovadora (Q/L) aumenta en 0.2607 puntos porcentuales. Estos resultados sugieren que las variables explicativas tienen un efecto positivo en la productividad de las firmas.

El panel B incluye la variable tecnologías de información (TI/L) y sus interacciones. Es importante acotar que sólo la interacción entre las TI/L y la gestión del conocimiento (GC/L) tiene un coeficiente positivo y significativo estadísticamente. Además, la variable tecnologías de información (TI/L) es significativa y positiva a un nivel de confianza del $95 \%$. Dicho de otra manera, el efecto de $\mathrm{Tl} / \mathrm{L}$ en la productividad depende positivamente de la tasa de crecimiento promedio de GC/L. El panel $\mathrm{C}$ representa el modelo completo con todas las variables y sus interacciones, tomando en consideración la implementación de efectos fijos en los agentes (firmas). 


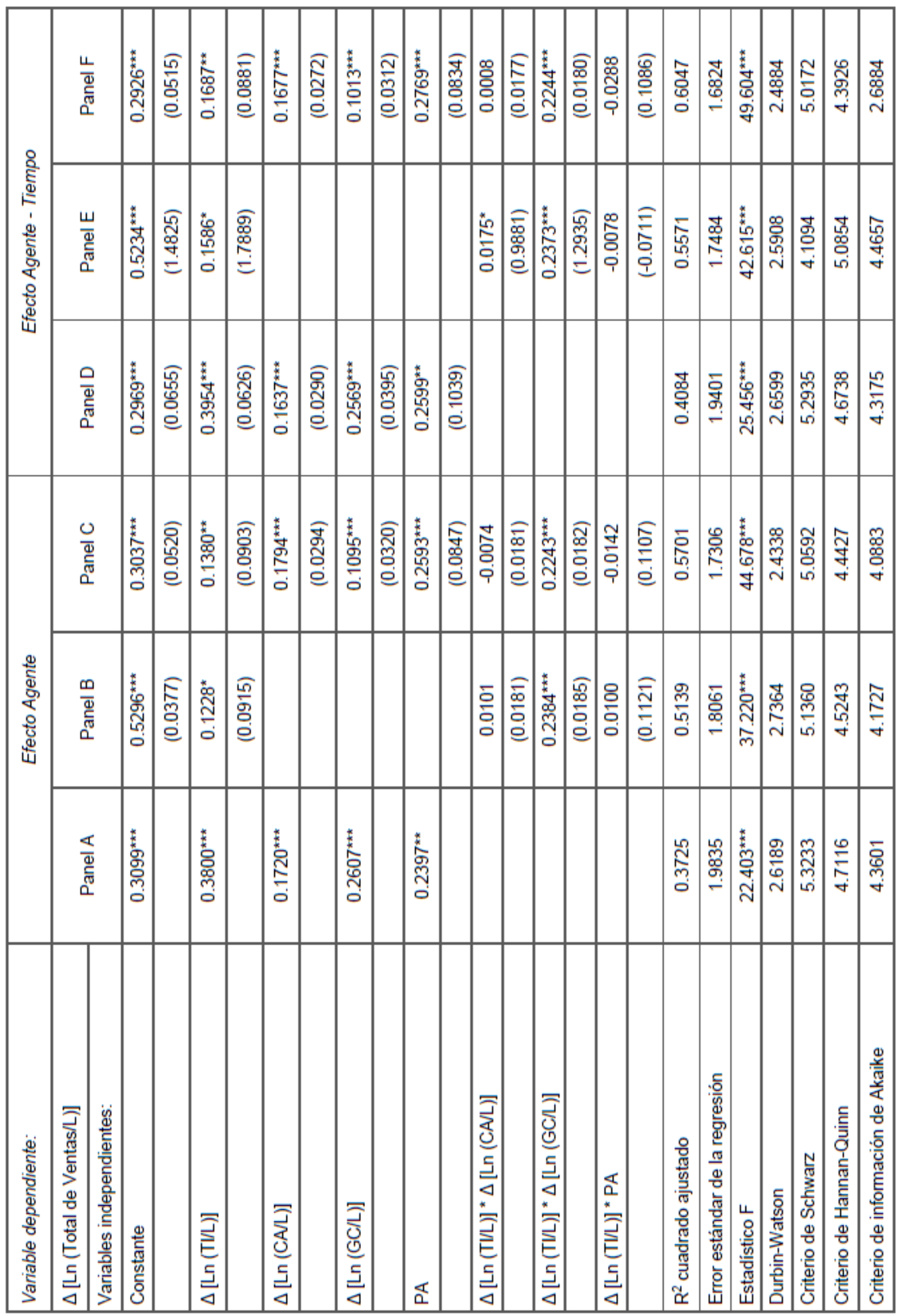


Individualmente, las variables explicativas inversión en tecnologías de información (TI), inversión en capacitación (CA), inversión en gestión del conocimiento (GC) y prácticas administrativas (PA) influyen positivamente en la productividad innovadora. Por otro lado, únicamente la interacción entre las tecnologías de información y la gestión del conocimiento (GC) influye en la productividad de las firmas, por lo que la variable GC cumple el rol de variable moderadora entre las TI y la productividad innovadora de las empresas de servicios. Al igual que el panel $\mathrm{B}$, el efecto de $\mathrm{TI} / \mathrm{L}$ en la productividad depende positivamente de la tasa crecimiento promedio de GC/L. En este escenario, evaluando la derivada parcial de la función de la productividad con respecto a $\mathrm{Tl} / \mathrm{L}$, determinada en la ecuación $\partial \Delta \ln Y / \partial \Delta \ln (T I / L)=\beta_{1}+\beta_{6}(G C / L)$, se concluye que un punto porcentual de $T \mathrm{Tl} / \mathrm{L}$ genera un incremento de 0.147 puntos porcentuales en la productividad innovadora de las firmas $(\mathrm{Q} / \mathrm{L})$, considerando que la tasa de crecimiento promedio de GC es de cuatro puntos porcentuales.

Los tres paneles restantes (D, E y F) reportan los resultados de las regresiones controladas por efectos fijos en los agentes y el tiempo. En el panel D, los coeficientes de las variables tecnologías de información (TI), inversión en capacitación (CA) e inversión en gestión de conocimiento (GC) son significativas al 99\% de confianza. También, la variable prácticas administrativas (PA) es positiva y significativa al $95 \%$ de confianza. En el panel E se incluyó la variable tecnologías de información (TI) y sus interacciones. La interacción entre las TI y la inversión en gestión del conocimiento (GC) y la interacción entre las tecnologías de información (TI) y la inversión en capacitación (CA) tienen coeficientes positivos y significativos estadísticamente. Esto indica que el efecto de $\mathrm{TI} / \mathrm{L}$ en la productividad innovadora depende de los procesos de mejora de la gestión del conocimiento y de la capacitación a los empleados de las firmas. Finalmente, el panel F representa el modelo completo con todas las variables y sus interacciones. Individualmente, se destaca que las variables tecnologías de información ( $\mathrm{TI})$, capacitación (CA), gestión del conocimiento (GC) y prácticas administrativas (PA) influyen positivamente en la productividad.

Avanzando en el análisis, únicamente el coeficiente de la interacción entre las tecnologías de información y la gestión del conocimiento (GC) es positivo y significativo, por lo que la variable GC cumple el rol de variable moderadora entre las TI y la productividad innovadora de las firmas de servicios. Se procedió, por ende, a evaluar la derivada parcial de la función de productividad con respecto a $\mathrm{TI} / \mathrm{L}$, de acuerdo a la siguiente expresión: $\partial \Delta \ln Y / \partial \Delta \ln (T I / L)=\beta_{1}+\beta_{6} \Delta \ln (G C / L)$. Bajo el efecto moderador de la tasa de crecimiento promedio de $\mathrm{GC} / \mathrm{L}$, que es de cuatro puntos porcentuales, se obtiene que el aumento de un punto porcentual de $\mathrm{TI} / \mathrm{L}$ genera un incremento de 0.178 puntos porcentuales en la productividad innovadora de las firmas (Q/L).

De acuerdo a los criterios de parsimonia (Akaike, Schwarz y Hannan-Quinn), los modelos que permiten hacer mejores estimaciones son los paneles C y F por presentar los valores más bajos en comparación con los demás paneles. Estos paneles tienen un coeficiente de determinación ajustado superior a 0.3 que es el límite general aceptado en la literatura (Baltagi, 2013; Semykina y Wooldridge, 2013), denotando que las variables consideradas en cada modelo explican un $57.01 \%$ y $60.47 \%$ de la variabilidad de la productividad innovadora, respectivamente. En resumen, los resultados sugieren que la integración de las tecnologías de información (TI) con la gestión del conocimiento produciría un efecto superior en la productividad innovadora, debido a que la variable gestión del conocimiento (GC) cumple un rol fundamental en el análisis de datos de panel con efectos fijos.

\section{Análisis en el largo plazo}

En esta última instancia se analiza el impacto que generan las tecnologías de información (TI) y las variables explicativas del estudio en la productividad y rentabilidad innovadora de las firmas en el largo plazo. La tabla 4 reporta un primer análisis a largo plazo únicamente para la inversión en tecnologías de información (TI) como variable principal del estudio con respecto a las variables endógenas de los modelos planteados. Los números en paréntesis reportan el valor del error estándar, los valores en corchete reportan el factor de inflación de la varianza y se consideraron niveles de significancia de $10 \%\left({ }^{*}\right), 5 \%\left({ }^{* *}\right)$ y $1 \%\left({ }^{* * *}\right)$. La variable dependiente de productividad innovadora se mide a través del siguiente operador: $\Delta$ [Ln (Total de Ventas/L)] 1016; mientras que la variable dependiente de rentabilidad innovadora se mide por medio del siguiente operador: $\Delta[\text { Ln (Utilidad Neta/L) }]_{10-16 .}$

Se aprecia que los parámetros estimados de las TI son positivos y significativos, evidenciando que estas generan un impacto importante tanto en la productividad como en la rentabilidad de las firmas de servicios en el largo plazo. En términos de elasticidad, las TI a largo plazo generan un aporte mayor en la productividad innovadora con 1.210 puntos porcentuales frente a 0.949 puntos porcentuales de incremento en la rentabilidad innovadora. Ambos modelos no presentaron problemas de autocorrelación, multicolinealidad y heterocedasticidad. Adicionalmente, la tabla 4 expone el efecto de las cuatro variables explicativas y sus interacciones con respecto a la productividad y rentabilidad innovadora de las firmas. La variable TI, en este nuevo análisis, mantiene parámetros significativos estadísticamente, demostrando que estas impactan positivamente importante en las variables endógenas en el largo plazo. 
Tabla 4: Análisis en el Largo Plazo de las Variables de Estudio en las Firmas de Servicios

\begin{tabular}{|c|c|c|c|c|c|c|}
\hline \multirow{2}{*}{ Variable } & \multicolumn{3}{|c|}{ Productividad Innovadora } & \multicolumn{3}{|c|}{ Rentabilidad Innovadora } \\
\hline & Modelo 1 & Modelo 2 & Modelo 3 & Modelo 4 & Modelo 5 & Modelo 6 \\
\hline \multirow[t]{2}{*}{ Constante } & $0.193^{* * *}$ & 0.060 & $0.066^{\star *}$ & $0.194^{* * *}$ & -0.008 & 0.000 \\
\hline & $(0.031)$ & $(0.056)$ & $(0.026)$ & $(0.028)$ & $(0.050)$ & $(0.044)$ \\
\hline \multirow[t]{3}{*}{$\Delta[\operatorname{Ln}(\mathrm{TI} / \mathrm{L})]_{10-16}$} & $1.210^{\star * \star}$ & $0.633^{* * *}$ & $0.628^{* \star *}$ & $0.949^{* * *}$ & $0.383^{\star \star \star}$ & $0.372^{\star * *}$ \\
\hline & $(0.053)$ & $(0.105)$ & $(0.095)$ & $(0.047)$ & $(0.094)$ & $(0.059)$ \\
\hline & & {$[6.380]$} & {$[5.271]$} & & [6.380] & [2.489] \\
\hline \multirow[t]{3}{*}{$\Delta[\operatorname{Ln}(\mathrm{CA} / \mathrm{L})]_{10-16}$} & & $0.238^{* * *}$ & $0.238^{* * *}$ & & $0.351^{* * *}$ & $0.367^{* * *}$ \\
\hline & & $(0,057)$ & $(0.057)$ & & $(0.051)$ & $(0.048)$ \\
\hline & & [1.827] & [1.827] & & [1.827] & [1.568] \\
\hline \multirow[t]{3}{*}{$\Delta[\operatorname{Ln}(\mathrm{GC} / \mathrm{L})]_{10-16}$} & & $0.438^{* * *}$ & $0.438^{* * *}$ & & $0.419^{* * *}$ & $0.406^{* * *}$ \\
\hline & & $(0.072)$ & $(0.072)$ & & $(0.065)$ & $(0.063)$ \\
\hline & & [2.269] & [2.269] & & [2.269] & {$[2.151]$} \\
\hline \multirow[t]{3}{*}{$\overline{\mathrm{PA}}_{10-16}$} & & 0.077 & & & $0.889^{*}$ & $0.812^{*}$ \\
\hline & & $(0.582)$ & & & $(0.524)$ & $(0.440)$ \\
\hline & & [1.413] & & & [1.413] & {$[1.000]$} \\
\hline \multirow[t]{3}{*}{$\Delta \operatorname{Ln}(\mathrm{TI} / \mathrm{L})]^{\star} \Delta[\operatorname{Ln}(\mathrm{CA} / \mathrm{L})]_{10-16}$} & & $0.027^{\star *}$ & $0.027^{\star *}$ & & 0.010 & \\
\hline & & $(0.013)$ & $(0.013)$ & & $(0.012)$ & \\
\hline & & [3.137] & [3.129] & & [3.137] & \\
\hline \multirow[t]{3}{*}{$\Delta \operatorname{Ln}(\mathrm{TI} / \mathrm{L})]^{\star} \Delta[\operatorname{Ln}(\mathrm{GC} / \mathrm{L})]_{10-16}$} & & $0.075^{\star * *}$ & $0.075^{\star \star \star}$ & & $0.041^{* * *}$ & $0.047^{* * *}$ \\
\hline & & $(0.017)$ & $(0.017)$ & & $(0.015)$ & $(0.013)$ \\
\hline & & {$[4,427]$} & [4.423] & & {$[4,427]$} & [3.200] \\
\hline \multirow[t]{3}{*}{$\Delta \operatorname{Ln}(\mathrm{TI} / \mathrm{L})]^{*} \overline{\mathrm{PA}}_{10-16}$} & & $0.275^{\star}$ & $0.265^{\star *}$ & & -0.036 & \\
\hline & & $(0.142)$ & $(0.119)$ & & $(0.128)$ & \\
\hline & & [5.444] & [3.853] & & [5.444] & \\
\hline $\mathrm{R}^{2}$ ajustado & 0.579 & 0.750 & 0.746 & 0.516 & 0.701 & 0.702 \\
\hline Estadístico F & $515.75^{\star \star \star}$ & $157.10^{* * *}$ & $183.78^{\star \star \star}$ & $400.03^{\star \star \star}$ & $126.17^{\star \star \star}$ & 177.07 \\
\hline Durbin-Watson & 1.935 & 1.961 & 1.960 & 1.964 & 2.042 & 2.034 \\
\hline
\end{tabular}

Los resultados indican que tanto la capacitación (CA) como la gestión del conocimiento (GC) poseen coeficientes positivos y significativos tanto para la productividad como para la rentabilidad innovadora, mientras que el coeficiente de las prácticas administrativas (PA) es significativo a un nivel de confianza de $90 \%$ únicamente en el modelo seis de rentabilidad, lo que denota una falta de integración de las tecnologías en información en la estructura organizacional de las empresas. Con respecto a la interacción de las TI con las variables explicativas, los coeficientes de las interacciones entre $\mathrm{Tl}^{*} \mathrm{CA}$ y $\mathrm{Tl}^{\star} \mathrm{GC}$ son significativos estadísticamente con respecto a la productividad y rentabilidad innovadora en largo plazo.

De esta forma, el modelo planteado demuestra que la variable gestión del conocimiento (GC) genera una incidencia importante en el crecimiento de las firmas de servicios en temas productivos y rentables, seguido de la inversión en capacitación en el largo plazo. Al igual que el caso anterior, ambos modelos no presentaron problemas de autocorrelación, multicolinealidad y heterocedasticidad. Un tercer modelo de productividad y rentabilidad fue creado a partir de las variables que presentaron coeficientes significativos estadísticamente. Se destaca que estos modelos explican el $74,6 \%$ y $70,2 \%$ de variabilidad de la productividad y rentabilidad innovadora, respectivamente. 


\section{Comprobación de Hipótesis y Hallazgos}

La tabla 5 resume los resultados de la comprobación de las hipótesis planteadas para el análisis de datos panel. Los resultados demuestran que la inversión en tecnologías de información influye positivamente en la productividad de las firmas de servicios. Igualmente se comprueba que la gestión del conocimiento cumple el rol de variable moderadora en la relación entre las TI y la productividad innovadora de las firmas en el corto plazo, asumiendo que las firmas de servicios que establecen procesos eficientes de investigación y desarrollo aumentan su productividad. En este contexto, la inversión en investigación y desarrollo representa un diferenciador relevante en el sector de servicios en países en vías de desarrollo, donde pocos son los sectores que están dispuestos a invertir y mejorar la gestión del conocimiento.

Tabla 5: Comprobación de Hipótesis con Datos de Panel (Agente y Agente-Tiempo)

\begin{tabular}{|c|c|c|c|c|}
\hline \multicolumn{5}{|c|}{ Agente - Tiempo } \\
\hline Hipótesis & Relación & Estimado & $p$ & Observación \\
\hline $\begin{array}{l}\mathrm{H}_{1} \text { : La implementación de las TI influye en la } \\
\text { productividad de las empresas de servicios }\end{array}$ & Significativa & 0.1687 & ** & Se acepta \\
\hline $\begin{array}{l}\mathrm{H}_{2 a} \text { : La inversión en capacitación cumple el rol de } \\
\text { variable moderadora entre la relación de la inversión } \\
\text { en } \mathrm{TI} \text { y la productividad de las empresas de servicios }\end{array}$ & No significativa & 0.0008 & - & Se rechaza \\
\hline $\begin{array}{l}\mathrm{H}_{2 b} \text { : La gestión del conocimiento cumple el rol de } \\
\text { variable moderadora entre la relación de la inversión } \\
\text { en } \mathrm{TI} \text { y la productividad de las empresas de servicios }\end{array}$ & Significativa & 0.2244 & $* * *$ & Se acepta \\
\hline $\begin{array}{l}\mathrm{H}_{2 c} \text { : Las prácticas administrativas cumple el rol de } \\
\text { variable moderadora entre la relación de la inversión } \\
\text { en } \mathrm{TI} \text { y la productividad de las empresas de servicios }\end{array}$ & No significativa & -0.0288 & - & Se rechaza \\
\hline
\end{tabular}

La tabla 6 reporta los resultados de la comprobación de las hipótesis planteadas para el análisis en el largo plazo. Los resultados demuestran que la inversión en tecnologías de información (TI) genera incidencia en la productividad y rentabilidad innovadora de las firmas en el largo plazo (Djellal y Gallouj, 2008). Por otro lado, la inversión en capacitación, la inversión en gestión del conocimiento y las prácticas administrativas cumplen el rol de variables moderadoras en la relación entre las TI y la productividad innovadora de las firmas en el largo plazo (Cao y Hoffman, 2011), comprobando que las empresas de servicios incrementan su productividad en el largo plazo cuando invierten de forma adecuada en investigación y desarrollo, capacitan a su personal e integran las $\mathrm{TI}$ a sus actividades administrativas y operacionales (Ortega et al., 2014).

Tabla 6: Comprobación de Hipótesis en el Largo Plazo

\begin{tabular}{|l|c|c|c|c|}
\hline \multicolumn{1}{|c|}{ Hipótesis } & Relación & Estimado & $p$ & Observación \\
\hline $\begin{array}{l}H_{1} \text { : La implementación de las TI influye en la productividad } \\
\text { de las empresas de servicios }\end{array}$ & Significativa & 0.628 & $* * *$ & Se acepta \\
\hline $\begin{array}{l}H_{2 a} \text { : La inversión en capacitación cumple el rol de variable } \\
\text { moderadora entre la relación de la inversión en TI y la } \\
\text { productividad de las empresas de servicios }\end{array}$ & Significativa & 0.027 & $* *$ & Se acepta \\
\hline $\begin{array}{l}H_{2 b} \text { : La gestión del conocimiento cumple el rol de variable } \\
\text { moderadora entre la relación de la inversión en TI y la } \\
\text { productividad de las empresas de servicios }\end{array}$ & Significativa & 0.075 & $* * *$ & Se acepta \\
\hline $\begin{array}{l}H_{20} \text { : Las prácticas administrativas cumple el rol de variable } \\
\text { moderadora entre la relación de la inversión en Tl y la } \\
\text { productividad de las empresas de servicios }\end{array}$ & Significativa & 0.265 & $* *$ & Se acepta \\
\hline $\begin{array}{l}H_{3}: \text { La inversión en TI influye en la rentabilidad de las } \\
\text { empresas de servicios }\end{array}$ & Significativa & 0.372 & $* * *$ & Se acepta \\
\hline
\end{tabular}


La presente investigación ofrece pruebas convincentes sobre la influencia positiva de las tecnologías de información (TI) en la productividad y rentabilidad innovadora de las firmas de servicios en el Ecuador, teniendo en cuenta el rol protagónico que cobran las TI en el corto y largo plazo. Esta validación es relevante dada las recomendaciones realizadas por varios investigadores (Aral et al., 2012; Arvanitis y Loukis, 2015; Biege et al., 2013; Brynjolfsson y Hitt, 1996; Djellal y Gallouj, 2008; Hawash y Lang, 2010; Ong y Chen, 2013; Zwick, 2003) que las tecnologías de información son un importante activo tangible para las organizaciones en los países desarrollados y una importante fuente de productividad y competitividad a través de su implementación en las firmas de los diversos sectores estratégicos de las naciones (Benitez-Amado y Walczuch, 2012; Bharadwaj, 2000; Orlikowski y lacono, 2001). Es por esta razón que dichos investigadores han sugerido realizar investigaciones de alto impacto en países en vías de desarrollo y poder determinar la influencia de las TI en la productividad, rentabilidad y desempeño de las firmas (Sabherwal y Jeyaraj, 2015).

\section{CONCLUSIONES}

De acuerdo al trabajo presentado y a los resultados obtenidos, se pueden plantear las siguientes conclusiones principales:

1. Los hallazgos obtenidos ofrecen nuevas evidencias empíricas que responden a la paradoja de la productividad y son congruentes con la revisión de literatura y fundamentación teórica de cada una de las variables del estudio; 2. Los resultados revelan que cada una de las variables explicativas, inversión en tecnologías de información, inversión en capacitación al personal, inversión en gestión del conocimiento y mejora de las prácticas administrativas de las firmas pueden llegar a presentar una influencia significativa y positiva en la productividad y rentabilidad de las firmas; 3 . El estudio ofrece un nuevo enfoque para la medición de la productividad, bajo el término de productividad innovadora que está asociada a resultados innovadores tangibles, producto de la inversión en TI y que provoca ventajas económicas a las empresas de servicios. Esta consiste en las ventas generadas por innovaciones y servicios nuevos en el mercado; eliminando así sesgos producidos por otros factores que aumentan la productividad; 4. La inversión en TI presentó un coeficiente positivo y significativo en el modelo de datos de panel con un nivel de confianza del $95 \%$, sin embargo, el efecto aumentó considerablemente en el largo plazo con un nivel de significancia de $99 \%$. Esto sugiere que toma un tiempo incorporar las tecnologías de información en la estructura organizacional, administrativa y operativa de las firmas; por tal motivo el efecto es más significativo en el largo plazo que en el corto plazo en términos de productividad innovadora en las organizaciones; 5 . Únicamente la inversión en gestión del conocimiento (GC) cumple el rol de variable moderadora en la relación entre las TI y productividad innovadora de las firmas en el análisis de datos de panel. Por otro lado, el análisis en el largo plazo demuestra que la inversión en capacitación (CA), inversión en gestión del conocimiento (GC) y las prácticas administrativas (PA) cumplen el rol de variables moderadoras en la relación entre las TI y la productividad, evidenciando que las empresas de servicios que establecen adecuadamente procesos de investigación y desarrollo, invierten en capacitación y entrenamiento del talento humano e integran las TI a sus prácticas administrativas en el largo plazo generan nuevos servicios y aumentan su productividad; 6 . El estudio también mostró evidencias de la relación positiva y significativa en el largo plazo entre la inversión en TI y la rentabilidad innovadora de las empresas de servicios; 7. A pesar de que el estudio actual proporciona evidencias de la influencia de las TI en la productividad, los resultados no son posibles de generalizar para los diversos sectores de la economía y en el contexto de otros países en vías de desarrollo.

Futuras líneas de investigación derivadas del actual estudio pueden incluir el análisis de otros sectores de la economía, como el manufacturero, de telecomunicaciones, etc., además de comparaciones entre países en vías de desarrollo y del primer mundo y observar la influencia de las TI en la productividad y rentabilidad de las empresas. De igual modo, futuras investigaciones pueden estar orientadas a cómo las TI impactan en variables de desempeño no financiero, tales como el índice de satisfacción interno en el uso y capacitación en manejo de las Tl y estudios que aborden la productividad administrativa y su impacto en la generación del servicio al cliente.

\section{REFERENCIAS}

Afflerbach, P., The Business Value of IT in Light of Prospect Theory: A New Explanation for IT Paradoxes, Business \& Information Systems Engineering, 57(5), 299-310 (2015)

Alavi, M. y D. Leidner, Knowledge Management and Knowledge Management Systems: Conceptual Foundations and Research Issues, MIS Quarterly, 25(1), 107-136 (2001)

Aral, S., E. Brynjolfsson y L. Wu, Three-Way Complementarities: Performance Pay, Human Resource Analytics, and Information Technology, Management Science, 58(5), 913-931 (2012)

Arévalo-Avecillas, D., C. Padilla-Lozano, M. Bustamante-Ubilla y C. Vidal-Silva, Contrastación de la Paradoja de la Productividad por el uso de las Tecnologías de Información: el Caso Ecuatoriano, doi: 10.4067/S071807642017000100017, Información Tecnológica, 28(1), 171-178 (2017) 
Arvanitis, S. y E. Loukis, Employee education, information and communication technologies, workplace organization, and trade: a comparative analysis of Greek and Swiss firms, Industrial and Corporate Change, 24(6), 1417-1442 (2015)

Arzola, M. y A. Mejias, Morfología del aprendizaje y la gestión del conocimiento del sector servicios de consultoría, Ingeniería Industrial, 37-51 (2010)

Baltagi, B., Econometric analysis of panel data, New York, John Wiley \& Sons (2013)

Banker, R. y R. Natajaram, Evaluating contextual variables affecting productivity using Data Envelopment Analysis, Operations Reseacrh, 56(1), 48-59 (2008)

Benitez-Amado, J. y R.M. Walczuch, Information Technology, the Organizational Capability of Proactive Corporate Environmental Strategy and Firm Performance: A Resource-Based Analysis, European J. of Infor. Systems, 21(6), 664-679 (2012)

Bertschek, I., D. Cerquera y G. Klein, More Bits - More Bucks? Measuring the Impact of Broadband Internet on Firm Performance, Information Economics and Policy, 25, 190-203 (2013)

Bharadwaj, A. S., A Resource-based perspective on information technology capability and firm performance: An empirical examination, MIS Quarterly, 24(1), 169-196 (2000)

Biege, S., G. Lay, T. Schmall y C. Zanker, Challenges of Measuring Service Productivity in Innovative, KnowledgeIntensive Business Services, Institute for Systems and Innovation Research, 1-20 (2013)

Black, S.E. y L. M. Lynch, How to Compete: The Impact of Workplace Practices and Information Technology on Productivity, The Review of Economics and Statistics, 83(3), 434-445 (2001)

Brynjolfsson, E. y L. Hitt, Paradox Lost? Firm-level evidence on the returns to information systems spending, Management Science, 4(42), 541-558 (1996)

Brynjolfsson, E. y L. Hitt, Computing productivity: Firm-level evidence, The Review of Economics and Statistics, 4(85), 793-808 (2003)

Cao, Q. y J.J. Hoffman, Alignment of Virtual Enterprise, Information Technology, and Performance, International Journal of Production Research, 49(4), 1127-1149 (2011)

Cardona, M., T. Kretschmer y T. Strobel, ICT and Productivity: Conclusions from the Empirical Literature, Information Economics and Policy, 25, 109-125 (2013)

Cefis, E. y M. Ciccarelli, Profit differentials and innovation, Economics of Innovation and New Technology, 14(1-2), 43-61 (2005)

Chang, Y.B. y V. Gurbaxani, Information Technology Outsourcing, Knowledge Transfer, and Firm Productivity, MIS Quarterly, 36(4), 1043-1063 (2012)

Charoensuk, S., W. Wongsurawat y D. B. Khang, Business-IT Alignment: A practical research approach, The Journal of High Technology Management Research, 25(1), 132-147 (2014)

Chen, J.-S. y H.-T. Tsou, Performance Effects of IT Capability, Service Process Innovation, and the Mediating Role of Customer Service, Journal of Engineering and Technology Management, 29(1), 71-94 (2012)

Chuang, S.-H., C. Liao, y S. Lin, Determinants of Knowledge Management with Information Technology support impact on Firm Performance, Information Technology Management, 14(3), 217-230 (2013)

Cobo, J. C., El Concepto de Tecnologías de la Información. Benchmarking sobre las definiciones de las TIC en la Sociedad del Conocimiento, Revista de Estudios de Comunicación, 295 - 318 (2009)

Darroch, J., y R. McNaughton, Examining the link between knowledge management practices and types of innovation, Journal of Intellectual Capital, 210-222 (2002)

Dedrick, J., y K. Kraemer, The Productivity Paradox: Is it Resolved? Is there a New One? What Does It All Mean for Managers? Center for Research on Information Technology and Organizations, 118, 1-13 (2001)

Dedrick, J., K. Kraemer y E. Shih, IT and Productivity in Developed and Developing Countries, Personal Computing Industry Center, 1-24 (2011)

Delone, W. y E. McLean, The DeLone and McLean Model of Information Systems Success: A Ten-Year Update, Journal of Management Information Systems, 19(4), 9-30 (2003)

Dewan, S. y K.L. Kraemer, Information technology and productivity: preliminary evidence from country-level data, Management Science, 4(46), 548-562 (2000)

Dhaene, G. y K. Jochmans, Likelihood Inference in an Autoregression with Fixed Effects, Econometric Theory, 32(1), $1178-1215(2016)$

Djellal, F. y F. Gallouj, Measuring and improving productivity in services. Issues, strategies and challenges, Edward Elgar Publications, 1-30 (2008)

Ghobakhloo, M., T.S. Hong, M.S. Sabouri y N. Zulkifli, Strategies for Successful Information Technology Adoption in Small and Medium Sized Enterprise, doi:10.3390/info3010036, Information, 36-67 (2012)

Han, C., y P.C. Phillips, First Difference Maximum Likelihood and Dynamic Panel Estimation, Journal of Econometrics, 175(1), 35-45 (2013) 
Hawash, R. y G. Lang, The Impact of Information technology on productivity in Developing Countries, Working Paper (2010) Hernández, R., C. Fernández y P. Baptista, Metodología de la Investigación Científica, México, Mc Graw-Hill (2010)

Hwang, J.-S., S. Kim y H. Lee, Breaking the Myths of the IT Productivity, KSII Transactions on Internet and Information Systems, 9(1), 466-482 (2015)

Ichniowski, C., K. Shaw y G. Prennushi, The Effects of Human Resource Management Practices on Productivity: A Study of Steel Finishing Lines, The American Economic Review, 87(3), 291-313 (1997)

Klomp, L. y G. Van Leeuwen, Linking innovation and firm performance: a new approach, International Journal of the Economics of Business, 8(3), 343-364 (2001)

Kraemer, K.L. y J. Dedrick, Information technology and productivity: results and policy implications of cross-country studies, Pohjola, Oxford University Press (2001)

Krugman, P. y M. Obstfeld, Economía internacional: Teoría y política, $7^{a}$ Ed., Madrid, Pearson Addison-Wesley (2006)

Kruiniger, H. Quasi ML Estimation of the Panel AR (1) Model with Arbitrary Initial Conditions, Journal of Econometrics, 173(1), 175-188 (2013)

Lantz, B., R. Andersson y P. Manfredsson, Preliminary Tests of Normality When Comparing Three Independent Samples, Journal of Modern Applied Statistical Methods, 15(2), 135-148 (2016)

Lapointe, L. y S. Rivard, Multilevel Model of Resistance to Information Technology Implementation, MIS Quarterly, 461491 (2005)

Licht, G. y D. Moch, Innovation and Information Technology in Services, Canadian J. of Economics, 32(2), $363-383$ (1999)

Lichtenberg, F.R., The Output Contribution of Computer Equipment and Personnel: A Firm-Level Analysis, Economics of Innovation and New Technology, 201-217 (1995)

Liu, H., W. Ke, K.K. Wei y Z. Hua, The Impact of IT Capabilities on Firm Performance: The Mediating Roles of Absorptive Capacity and Supply Chain Agility, Decision Support Systems, 54(3), 1452-1462 (2013)

Malhotra, N.K., Investigación de Mercados, México D.F., Pearson (2008)

Marulanda, C., J. Giraldo y M. López, Evaluación de la Gestión del Conocimiento en las Organizaciones de la Red de Tecnologías de Información y Comunicaciones del Eje Cafetero en Colombia, doi: 10.4067/S0718-07642013000400012, Información Tecnológica, 24(4), 105-116 (2013)

Melville, N., Impact of IT investment: An industry analysis. Irvine: Centre for Research on Information Technology and Organization, University of California (2001)

Ong, C. y P. Chen, Information Technology Capability-Enabled Performance, Future Performance, and Value, Industrial Management \& Data Systems, 113(5), 669-682 (2013)

Organización Mundial del Comercio, World Trade Statistical Review 2017, Geneva, WTO (2017)

Orlikowski, W.J. y C.S. lacono, Desperately seeking the 'IT' in IT research - A call to theorizing the IT artifact, Information Systems Research, 121-134 (2001)

Ortega, R., M. Piva y M. Vivarelli, The transatlantic productivity gap: Is R\&D the main culprit? Canadian Journal of Economics, 47(4), 1342-1371 (2014)

Peng, J., J. Quan, G. Zhang y A. Dubinsky, J. Mediation effect of business process and supply chain management capabilities on the impact of IT on firm performance: Evidence from Chinese firms, International Journal of Information Management, 36(1), 89-96 186 (2016)

Pérez, C. y C.P. López, Econometría avanzada, técnicas y herramientas, Madrid, Pearson Prentice Hall (2008)

Pierce, L., D. Snow y A. McAfee, Cleaning house: The impact of information technology monitoring on employee theft and productivity, Management Science, 61(10), 2299-2319 (2015)

Rawley, E. y T.S. Simcoe, Information technology, productivity, and asset ownership: Evidence from taxicab fleets, Organization Science, 24(3), 831-845 (2013)

Sabherwal, R. y A. Jeyaraj, Information technology impacts on firm performance: an extension of Kohli and Devaraj (2003), MIS Quarterly, 39(4), 809-836 (2015)

Semykina, A. y J. M. Wooldridge, Estimation of dynamic panel data models with sample selection, Journal of Applied Econometrics, 28(1), 47-61 (2013)

Tallon, P., K. Kraemer y V. Gurbaxani, Executives' Perceptions of the Business Value of Information Technology: A Process-Oriented Approach, Journal of Management Information Systems, 16(4), 145-173 (2000)

World Economic Forum, The Global Competitiveness Report 2014-2015, Ginebra, World Economic Forum, Recuperado el 2015 de: The Global Information Technology Report (2014)

Zwick, T., The Impact of ICT Investment on Establishment Productivity, Centre for European Economic Research, 1-20 (2003) 